- Frequency and length of time for delayed cord clamping.

- Time for umbilical line placement, colostrum administration, and parent updates.

The team presents the results through multiple forums including biannual 'Awareness days' which are a fun way to update and educate staff using presentations, competitions, simulations and bite-sized teaching. They are a platform for launching new initiatives and equipment and are accompanied by social media updates and emails for staff unable to attend.

In December 2018 the PQoC group produced a lanyard card to help staff deliver more consistent care pre, during and post delivery.

In October 2019, we have launched a re-useable 'Checklist' to be used at delivery with the aim to improve documentation and adherence to the guideline. If the guideline was not adhered to or documentation is incomplete, the care will be discussed with the team involved.

Results The lanyard card was received very positively. 69\% of colleagues reported that the lanyard card had changed the care they had provided to a preterm admission. In particular, the medical staff commented on how useful it is to have a prompt card to brief the team pre delivery and use for teaching. The nursing team reported feeling more confident at setting up the bedspace and getting umbilical lines ready. There was an improvement seen in the percentage of babies who received delayed cord clamping from 38\% to 55\% and the average time from 45 to 53 seconds. However, there were not significant improvements in other audited areas.

We are awaiting results from the introduction of the 'Checklist'.

Conclusion The PQoC group are very passionate about providing preterm babies with optimal care from birth. We continuously strive to improve care through creating and evaluating different initiatives.

\section{G120(P) ABSTRACT WITHDRAWN}

\section{G121(P) THINK MAGNESIUM: CONCERTED MULTIDISCIPLINARY IMPROVEMENT INITIATIVE TO INCREASE ANTENTANAL MAGNESIUM UPTAKE IN < $\mathbf{3 0}$ WEEK PRETERM BIRTHS}

${ }^{1} \mathrm{CS}$ Narayanan, ${ }^{1} \mathrm{~A}$ Katana, ${ }^{2} \mathrm{H}$ Abdulle, ${ }^{2} \mathrm{M}$ Coker, ${ }^{2} \mathrm{D}$ Mitra, ${ }^{2} \mathrm{~N}$ Shetty. ${ }^{1}$ Department of Neonatology, Watford General Hospital, UK; ${ }^{2}$ Department of Obstetrics and Gynaecology, Watford General Hospital, UK

\subsection{6/archdischild-2020-rcpch.96}

Aim There is compelling evidence that magnesium sulfate (MgSO4) given antenatally in threatened preterm labour is neuroprotective and reduces risk of cerebral palsy in offspring. National Neonatal Audit Programme (NNAP) in the UK monitors compliance to antenatal magnesium uptake in births < 30 weeks. Our centre was a negative outlier (16\% vs $43 \%$ national average) for this audit measure in the 2016. Our aim was to improve $\mathrm{MgSO} 4$ compliance by at least 50\% within the first year and then to sustain achieved improvement.

Methods A multidisciplinary improvement team comprising of neonatologists, obstetricians and midwives was formed. This team process mapped a preterm mother's admission to understand enablers and barriers to timely $\mathrm{MgSO} 4$ administration.
A driver diagram conceptualised overall aim, primary \& secondary drivers to inform change ideas. Change ideas (MgSO4 awareness sessions, clear guideline, preterm labour proforma, Think Mg posters, ward level champions, daily safety huddles, monthly display of compliance figures) were tested in iterative PDSA cycles. Number of preterm births $<30$ weeks between a non-compliant episode was plotted on a $\mathrm{g}$ chart which displayed performance over time.

Results In 2016, only 3/20 (15\%) preterm mothers received antenatal MgSO4. With improvement efforts and monitoring via PDSA cycles and $\mathrm{g}$ charts we saw a steady increase in MgSO4 uptake. In 2017 10/18 (56\%) and in 2018, 14/18 (77\%) received antenatal $\mathrm{MgSO} 4$. Opportunities between events ' $g$ ' chart tracked number of eligible births between a noncompliant episode. There were a few deliveries with short arrival to delivery intervals in late 2018, with drop in our compliance. Further analysis of these cases highlighted certain delays in triage ward, and they were addressed. Overall, in a 3 -year period $\mathrm{MgSO} 4$ compliance rose from $16 \%$ to $77 \%$. We also involved service users, by providing a parental information leaflet and including a check box in golden hour care checklist.

Conclusion Using a well-structured quality improvement plan, we were able to understand the barriers to $\mathrm{MgSO} 4$ use and systematically introduce changes that resulted in improved compliance. Collaborative working between multidisciplinary staff across specialties was key to our success and we hope to further scale up and sustain the improvement.

\section{G122(P) ABSTRACT WITHDRAWN}

\section{G123(P) REAL TIME RESOURCE LOCATOR FOR ACUTE NEONATAL TRANSFER SERVICE (ANTS): LOCANTS, A COMBINED TOOL AND SYSTEM FOR EFFICIENCY GAINS IN ACUTE NEONATAL TRANSFER}

${ }^{1} \mathrm{~A}$ Dhar, ${ }^{2} \mathrm{~S}$ Rattigan, ${ }^{3} \mathrm{E}$ Clarke, ${ }^{2} \mathrm{~L}$ Barford, ${ }^{4} \mathrm{R}$ Chatterjee, ${ }^{1} \mathrm{~S}$ Broster. ${ }^{1}$ Neonatal Transfer and NICU, Acute Neonatal Transfer Service and Addenbrookes Hospital, Cambridge, UK; ${ }^{2}$ Neonatal Transfer, Acute Neonatal Transfer Service, Cambridge, UK; ${ }^{3}$ Woman and Childrens' Services, Addenbrookes Hospital, Cambridge, UK; ${ }^{4}$ Cloud Data Specialist, Google, London, UK

\subsection{6/archdischild-2020-rcpch.97}

Aims Technological improvement remains a constant need in neonatal transport. ANTS is responsible for a large geographical area ( 6 counties, $15 \%$ of UK) with a large population density (>68,000 deliveries/yr, $>6.168$ million people). 13 transfer services out of 14 in UK are unable to meet nationally agreed key performance indicator (KPI) of mobilisation (60 min) for time critical transfers. Time taken to complete the clinical referral, collating and checking all resources (drugs, vehicle etc.), availability of teams are the main contributory factors for this delay. To improve the efficiency of transport service we are developing a tool, LocANTS ; to validate this tool we introduced a small part of this as a manual version (paper/screen) which is updated daily at 08:00 to localise available resources from January, 2019.

Method The paper copy includes on-call team/shift, pending transfers, vehicle, equipment, stock medication related updates, road and weather conditions, regional cot status, review of 
transfers (for shared learning) and in-utero transfers in the previous 24 hrs.

Results Data from 4 months showed a reduction of mean dispatch time for emergency transfers (including time critical) from 39 to $35 \mathrm{~min}$ (prior to the introduction of automation). As part of the shared learning component, 14 cases were identified and discussed with referring hospitals over the 4 months compared to $3,4,3$ cases in each of the previous year's respectively. The mean time of return of equipment after repair reduced from 31.3 to 9.8 days.

Conclusion By locating some available resources at a single point of time we observed positive changes in a number of measurable parameters. With this validation now we are building a dynamic real time platform (LocANTS) as proof of concept to show all the available resources ( including clinical parameters ) required for neonatal transfer in one consolidated screen alongside videoconferencing replacing the current audioconference, so that the team are ready to despatch 'time to roll' immediately the call for a transfer is made which will significantly improve KPI, handover quality, shared learning opportunities across the region, leading to efficient improved patient care.

\section{G124(P) JUNIOR DOCTORS AS QUALITY AND SAFETY LEADS}

A Yassaee, T Hodder, M Alam. Neonatal Department, Newham University Hospital, Barts Health, London, UK

\subsection{6/archdischild-2020-rcpch.98}

Aims Improve departmental approach to quality and safety (Q $+S$ ) issues, reporting culture and frontline staff's engagement with quality improvement through appointment of junior doctor $\mathrm{Q}+\mathrm{S}$ leads.

Methods Two junior doctors used the Model for Improvement. Cycle 1: Creating an issues log on the bottom of the handover list, enabling any member of the clinical team to $\mathrm{Q}$ $+\mathrm{S}$ issues. New items were discussed during handover. Issues included significant events, Datixes, and positive episodes which warranted promotion. Cycle 2: Introducing a weekly, minuted departmental meeting, chaired by a trainee. The meeting ran through the issue log, discussed complex cases, and would highlight opportunities for quality improvement. Cycle 3: Creating an online Greatix portal to encourage staff to highlight good practice, coupled with a monthly newsletter. Cycle 4: Formalising the role across changeover in order to ensure sustainability. This included a formal job description, advertisements to incoming trainees and negotiating protected time for individual(s) to carry out the role.

Results The weekly meetings supported multidisciplinary working and promoted the discussion of quality and safety issues in everyday clinical activities. The role has brought significant benefit the department's functioning and it was felt a good use of resource to offer protected time to an incoming trainee. There was good engagement with the Greatix system with 30 submitted and 3 newsletters produced. As a result of the structured approach in the weekly meetings, other trainees felt empowered to lead on their own quality improvement projects, including work with maternity and pathology services. Due to their knowledge, the $\mathrm{Q}+\mathrm{S}$ leads would often be asked to represent the department at governance/management meetings and their suggestions have led to projects in other departments.
Conclusion Junior doctors can be effective $Q+S$ advocates and leaders. Senior buy-in is essential for trainees to flourish in this role. Experience or training in leadership and/or QI is beneficial.

\section{G125(P) PASSING THE BATON: IMPROVING COMPETENCE AND CONFIDENCE IN HANDING OVER}

${ }^{1} \mathrm{D}$ Roberts, ${ }^{2} \mathrm{C}$ Lethaby. 'Department of Paediatric Medicine, Leeds Children's Hospital, Leeds, UK; ${ }^{2}$ Department of Paediatric Haematology and Oncology, Leeds Children's Hospital, Leeds, UK

\subsection{6/archdischild-2020-rcpch.99}

Introduction Clinical handover is crucial for safe, effective care of patients, and an opportunity for staff development and team building. A survey of medical staff in a tertiary children's hospital revealed that whilst 60\% have received structured training or feedback on effective handover at some point in their career, only $41 \%$ felt they received adequate training to hand over effectively. Confidence levels were rated on average at 53\%, and respondents recognised a need for further training.

Aims Improve competence and confidence of paediatric staff in handing over.

Methods Two structured, facilitator-led, interactive, group training sessions were developed and offered to paediatric staff utilising 'Plan, Do, Study, Act' (PDSA) cycles. Participants covered material on published handover guidelines, completed focused tasks designed to develop key skills which underpin handover (e.g. distilling pertinent information from case notes, prioritising workload), and drew these together to deliver structured written and verbal handovers using a validated tool (Situation, Background, Assessment, Recommendation [SBAR]). Guided discussion and reflection were promoted, and personalised verbal feedback given to participants.

Results A total of 28 attendances were recorded across 26 members of staff including junior doctors, advanced nurse practitioners, and physician associates. Anonymous feedback was sought from participants on the quality of training. Following the sessions $94.4 \%$ and $100 \%$ of attendees, respectively, who responded either agreed or strongly agreed that they felt more confident in the subject of handing over. Participants identified good aspects of training as it being practical, interactive, realistic, not critical, and encouraging. Challenges included having sufficient protected time for staff to attend and tailoring the sessions to the experience and seniority of attendees.

Conclusion This project has shown that structured, interactive training sessions have scope to increase the confidence of paediatric medical staff in handing over. Training requires that facilitators and participants have adequate, protected time to attend, and may, therefore, warrant inclusion in regional training days. Staff will be surveyed within six months to assess the longer-term impact of these training sessions on competence.

\section{G126(P) ABSTRACT WITHDRAWN}

